\title{
Activation of transposable elements and genetic instability during long-term culture of the human fungal pathogen Candida albicans
}

\author{
Leszek Potocki • Ewelina Kuna • Kamila Filip • Beata Kasprzyk • \\ Anna Lewinska $\cdot$ Maciej Wnuk
}

Received: 4 February 2019/ Accepted: 8 April 2019/Published online: 15 April 2019

(C) The Author(s) 2019

\begin{abstract}
It has been repeatedly reported that transposable elements (TE) become active and/or mobile in the genomes of replicatively and stress-induced senescent mammalian cells. However, the biological role of senescence-associated transposon activation and its occurrence and relevance in other eukaryotic cells remain to be elucidated. In the present study, Candida albicans, a prevalent opportunistic fungal pathogen in humans, was used to analyze changes in gene copy number of selected TE, namely Cirt2, Moa and Cmut1 during long-term culture (up to 90 days). The effects of stress stimuli (fluconazole, hydrogen peroxide, hypochlorite) and ploidy state (haploid, diploid, tetraploid cells) were also considered. An increase in copy number of Cirt2 and Moa was the most accented in tetraploid cells after 90 days of culture that was accompanied by changes in karyotype patterns and slightly more limited growth rate compared to haploid and diploid cells. Stress stimuli did
\end{abstract}

L. Potocki - E. Kuna - K. Filip · B. Kasprzyk ·

M. Wnuk ( $\square)$

Department of Genetics, Faculty of Biotechnology,

University of Rzeszow, Pigonia 1, 35-310 Rzeszow,

Poland

e-mail: mawnuk@gmail.com

A. Lewinska ( $\square)$

Department of Cell Biochemistry, Faculty of

Biotechnology, University of Rzeszow, Pigonia 1,

35-310 Rzeszow, Poland

e-mail: alewinska@o2.pl not potentiate TE activity. Elevation in chromosomal DNA breaks was also observed during long-term culture of cells of different ploidy, however this was not correlated with increased TE activity. Our results suggest that increased TE activity may promote genomic diversity and plasticity, and cellular heterogeneity during long-term culture of $C$. albicans cells.

Keywords Candida albicans · Long-term culture · Transposon activity $\cdot$ Karyotype profiling - DNA breaks $\cdot$ Stress inductors

\section{Introduction}

The budding yeast Saccharomyces cerevisiae has become the most popular unicellular and genetically tractable aging model system, playing a key role in the discovery of Sir2, TOR, RAS, adenylate cyclase, PKA and S6 kinase as conserved modulators of longevity in eukaryotes (Kaeberlein 2010; Longo et al. 2012). In general, there are two assays for yeast aging, namely the evaluation of replicative lifespan (RLS) and chronological lifespan (CLS) (Kaeberlein 2010; Longo et al. 2012). RLS measures the replicative potential of the cell, i.e. the number of daughter cells produced by a mother cell before senescence (Mortimer and Johnston 1959), whereas CLS measures the survival of a non-dividing population in a stationary 
culture (Fabrizio and Longo 2003). More recently, a new distinctive microbial model for cellular aging has been established, namely Candida albicans, a polymorphic fungus and a prevalent opportunistic fungal pathogen in humans (Fu et al. 2008). Both RLS as well as CLS assays have been adapted to $C$. albicans aging studies (Fu et al. 2008; Chen et al. 2012; Lin and Austriaco 2014). C. albicans can switch between two distinct morphological states, namely a yeast-like form (blastospore) and a filamentous form (hyphae) that can be modulated by nutrient composition in a culture medium, $\mathrm{pH}$ or temperature. Smaller replicatively young yeast form (daughters) and replicatively old hyphae (mothers) can be separated by centrifugation on a sucrose gradient that, in contrast to $S$. cerevisiae RLS, allows for more efficient large-scale isolation of old cells and may facilitate biochemical characterization and genomics/proteomics studies of cellular aging ( $\mathrm{Fu}$ et al. 2008). Similarly to $S$. cerevisiae, replicatively old $C$. albicans cells have been shown to accumulate glycogen and oxidatively damaged proteins (Fu et al. 2008). Moreover, deletion of the SIR2 gene resulted in decreased RLS, while insertion of an extra copy of SIR 2 extended RLS that indicate that Sir2 is also a regulator of cellular aging in C. albicans (Fu et al. 2008). It has been reported that CLS of C. albicans could be also extended by reducing the concentration of glucose from $2 \%$ to $0.5 \%$ in synthetic complete (SC) medium (calorie restriction conditions) that has been previously observed during chronological aging in S. cerevisiae (Chen et al. 2012). Moreover, $C$ albicans as a Crabtree negative fungus that prefers respiration to fermentation even in the presence of glucose may be considered as a good model for providing complimentary comparisons to aging and calorie restriction studies in a Crabtree positive S. cerevisiae (Lin and Austriaco 2014).

In general, two classes of transposable elements (TEs) can be distinguished, namely class I elements (copy-and-paste retrotransposons) that utilize reverse transcribed RNA intermediates to produce copies of themselves and class II elements (cut-and-paste DNA transposons) that excise from a donor site to reintegrate elsewhere in the genome (Wicker et al. 2007; Burns 2017). It has been suggested that more than half of human DNA is comprised of interspersed repeats resulting from replicative copy and paste events of retrotransposons (Burns and Boeke 2012). Altered expression of transposable elements can drive mutations in tumorigenesis and can be considered as a hallmark of cancer (Burns 2017). More recently, activation of transposable elements has been also documented in replicatively and stress-induced senescent human cells as well as during normal aging in mammalian somatic tissues (De Cecco et al. 2013a, b; Colombo et al. 2018; De Cecco et al. 2019). However, little is known about the biological function(s) of ageassociated increase in TE activity and related mechanisms. Age-mediated changes in the mobilomes of lower eukaryotes and non-mammalian systems, especially in well-established model organisms, and their consequences also have been poorly addressed.

The aim of the present study was to investigate the changes in the copy number of selected TEs (Cirt2, Moa and Cmut1) during long-term culture of $C$. albicans cells of different ploidy (haploid, diploid and tetraploid cells) in control conditions as well as after treatment with stress stimuli (fluconazole, hydrogen peroxide, hypochlorite), and their effects on growth rate, cell viability, karyotype patterns and genetic instability. We have developed an experimental protocol for a long-term culture of $C$. albicans cells at a high density in a rich and fresh YPD medium that mimicked the survival of a non-dividing population in a stationary culture in synthetic complete (SC) medium (Fabrizio and Longo 2003). However, as a spent medium has been replaced by a fresh one every 2 days of 90 days of culture, the impact of acidification of the culture medium, the phenomenon of accumulation of acetic acid in SC spent medium during chronological aging in yeast (Burtner et al. 2009), on C. albicans cell viability was minimized as well as starvation and related nutritional stress responses were limited. We have shown for the first time that TE activity is elevated during long-term culture of $C$. albicans cells that is accompanied by changes in karyotype profiles and may in turn promote genomic diversity and cellular heterogeneity as an adaptive response.

\section{Materials and methods}

Strains and culture conditions

The following Candida albicans strains were used: 302 (haploid), SC5314 (diploid) and T15 (FH6, tetraploid but trisomic for chromosomes $2 / 3$ with 
multiple copies of chromosome 5L) (Selmecki et al. 2008). The strains were a generous gift from Prof. Judith Berman (Department of Molecular Microbiology and Biotechnology, Tel Aviv University, Israel).

C. albicans cells from one single colony were routinely cultured on liquid yeast extract peptone dextrose (YPD) medium (1\% w/v Difco Yeast Extract, $2 \% \mathrm{w} / \mathrm{v}$ Difco Yeast Bacto-Peptone, $2 \% \mathrm{w} / \mathrm{v}$ dextrose) (BD Biosciences, Sparks, MD, USA) with shaking at $28{ }^{\circ} \mathrm{C}$. For long-term cultures during stress conditions, several stress stimuli were considered, namely fluconazole (100 ng/ml, Sigma-Aldrich, Poznan, Poland), hydrogen peroxide ( $2 \mathrm{mM}$, Sigma-Aldrich, Poznan, Poland) and sodium hypochlorite $(5 \mathrm{mM}$, Sigma-Aldrich, Poznan, Poland). The concentrations of stress inductors were selected on the basis of $\mathrm{IC}_{50}$ values. For long-term cultures, cells at the logarithmic phase of growth were suspended at $1 \times 10^{8}$ cells $/ \mathrm{ml}$ in YPD medium with or without stress stimuli and cultured with shaking at $28{ }^{\circ} \mathrm{C}$. During the first 4 days of long-term culture, fresh solutions of tested agents were added every $12 \mathrm{~h}$ and then stress stimuli were removed and culture was continued for up to 90 days with medium change every $48 \mathrm{~h}$. After 14, 28 and 90 days of culture, samples were taken for the evaluation of prolonged effects of stress stimuli after their removal from the medium.

Growth rate and cell viability

After 14, 28 and 90 days of culture, growth rate was analyzed as previously described (Lewinska et al. 2011). Briefly, cells were removed, washed, suspended in YPD medium (a total volume of $150 \mu \mathrm{l}$ with working concentration of $5 \times 10^{6}$ cells $/ \mathrm{ml}$ per well was considered) and cultured in a 96-well format incubator with shaking at $28{ }^{\circ} \mathrm{C}$ for $8 \mathrm{~h}$. Cell growth was monitored turbidimetrically at $600 \mathrm{~nm}$ in a Tecan microplate reader every $1 \mathrm{~h}$ during a $8 \mathrm{~h}$ period.

After 14, 28 and 90 days of culture, cell viability was analyzed as previously described (Lewinska et al. 2014a). Briefly, LIVE/DEAD ${ }^{\circledR}$ Yeast Viability Kit (Thermo Fisher Scientific, Warsaw, Poland) was used according to the manufacturer's instructions. Cells were washed and stained with a mixture of $\mathrm{FUN}^{\circledR} 1$ and Calcofluor ${ }^{\circledR}$ White M2R and inspected under an Olympus BX61 fluorescence microscope equipped with a DP72 CCD camera and Olympus CellF software. A total of 200 cells were used for the analysis.

Cell morphology analysis using imaging flow cytometry

Candida cell morphology (shape) was investigated using Amnis ${ }^{\circledR}$ FlowSight ${ }^{\circledR}$ imaging flow cytometer and IDEAS software version 6.2.187.0 (Merck Millipore, Warsaw, Poland). Two subpopulations of cells were considered, namely spherical cells (circularity) and non-spherical cells (non-circularity). Representative histograms and cell images are presented. The parameter circularity object was analyzed and normalized frequency was plotted against circularity object. 5000 cells per sample triplicate were analyzed. The percentage of cells of non-spherical cell subpopulation is presented.

\section{Cell size analysis}

Digital images of Candida cells were captured using an Olympus BX61 fluorescence microscope equipped with a DP72 CCD camera and Olympus CellF software (Olympus, Warsaw, Poland). ImageJ software (http://rsbweb.nih.gov/ij/) was used to analyze the cell size. Cell size was expressed as arbitrary units [a.u.].

\section{DNA content analysis}

Candida cells were fixed and stained as comprehensively described elsewhwere (Potocki et al. 2019). Some minor modifications were provided, namely fixed cells were treated with $5 \mathrm{mg} / \mathrm{ml}$ proteinase $\mathrm{K}$ for better staining performance. Briefly, for DNA visualization, cells were counterstained with a drop of mounting medium containing $4^{\prime}, 6^{\prime}$-diamino-2phenylindole (DAPI) (Cambio, Cambridge, UK) and then analyzed using an Olympus BX61 fluorescence microscope equipped with a DP72 CCD camera and Olympus CellF software (Olympus, Warsaw, Poland). The CCD capture conditions were as the following: exposure time $150 \mathrm{~ms}, 100 \mathrm{x}$ oil immersion objective. DAPI fluorescent signals were collected using DAPI filters $\left(\lambda_{\mathrm{ex}}=345 \mathrm{~nm}, \lambda_{\mathrm{em}}=455\right)$. Fluorescence microscopy was adapted for DNA content analysis (Potocki et al. 2019). ImageJ software (http://rsbweb. 
nih.gov/ij/) was used to analyze the nuclear DNA content. DNA content was expressed as arbitrary units [a.u.].

\section{Karyotype profiling}

DNA plugs were obtained using BIORAD CHEF Yeast Genomic DNA Plug Kit using a standard protocol (Selmecki et al. 2010) according to the manufacturer's instructions. Contour clamped homogeneous electric field (CHEF)—pulsed-field gel electrophoresis (PFGE) separation of $C$. albicans chromosomes was performed on a $1 \%$ agarose gel in $0.8 \times$ TBE according to the manufacturer's instructions using CHEF-DR ${ }^{\circledR}$ III Pulsed Field Electrophoresis System (Biorad, Warsaw, Poland) and the following conditions: 60 to $120 \mathrm{~s}$ switch, $6 \mathrm{~V} \times$ $\mathrm{cm}^{-1}, 120^{\circ}$ angle for $36 \mathrm{~h}$, followed by 120 to $300 \mathrm{~s}$ switch, $4.5 \mathrm{~V} \times \mathrm{cm}^{-1}, 120^{\circ}$ angle for $12 \mathrm{~h}$. After CHEF-PFGE separation, $C$. albicans chromosomes were stained using ethidium bromide staining.

Detection of transposable elements (TE): Southern blot analysis

First, several TE probes were created. The following oligonucleotide primer pairs for TE sequences, namely Cmut1, Cirt2 and Moa were designed using NCBI database:

- Cmut1: CAATAGCCACGACTTGCAGA, TCAG ATAAATTGCTCGCATGA;

- Cirt2: TGgtcGatatgaAgtgGCtatg, ATt GTTGACACCCCACGACT;

- Moa: GTCGTGGAGTACGACGTtATCA, GTC GTGTAGTAGCAACACTTCG.

Amplification was performed in $50 \mu \mathrm{l}$ aliquots of a solution containing $5 \mu \mathrm{l}$ of $10 \times$ PCR buffer with $\mathrm{MgCl}_{2}, 1.75 \mu \mathrm{l}$ of $10 \mathrm{mM}$ dNTPs, $300 \mathrm{nM}$ primers and $3.5 \mathrm{U}$ of Expand Long Template Enzyme Mix using Expand Long Template PCR System (SigmaAldrich, Poznan, Poland). The PCR reaction was performed in Mastercycler Eppendorf using the following conditions: initial denaturation at $94{ }^{\circ} \mathrm{C}$ for $2 \mathrm{~min}$, followed by 10 cycles of denaturation at
$94{ }^{\circ} \mathrm{C}$ for $10 \mathrm{~s}$, annealing at $45^{\circ} \mathrm{C}$ for $30 \mathrm{~s}$, elongation at $68{ }^{\circ} \mathrm{C}$ for $2 \mathrm{~min}$, and followed by 25 cycles of denaturation at $94{ }^{\circ} \mathrm{C}$ for $15 \mathrm{~s}$, annealing at $52{ }^{\circ} \mathrm{C}$ for $30 \mathrm{~s}$, elongation at $68{ }^{\circ} \mathrm{C}$ for $2 \mathrm{~min}$ and a final extension step at $68{ }^{\circ} \mathrm{C}$ for $7 \mathrm{~min}$. Purified PCR products were labeled using the labeling kit DIG High Prime (Roche, Warsaw, Poland). Briefly, $1 \mu \mathrm{g}$ DNA (a final volume of $16 \mu \mathrm{l}$ ) was denatured at $95{ }^{\circ} \mathrm{C}$ for 10 min and DNA was then labeled with $4 \mu \mathrm{l}$ of digoxygenin-11-dUTP. Labeling reaction was performed overnight at $37{ }^{\circ} \mathrm{C}$ and then stopped by heating the samples at $65^{\circ} \mathrm{C}$ for $10 \mathrm{~min}$.

Second, transposable elements were detected using Southern blot analysis. After PFGE separation, $C$. albicans chromosomes were transferred onto a nylon membrane (Roche, Warsaw, Poland) by capillary transfer. Then, the membrane was hybridized to digoxigenin (DIG)-labeled TE-specific probes at $42{ }^{\circ} \mathrm{C}$ for $24 \mathrm{~h}$ and transposable elements (Cmut1, Cirt2, Moa) were detected using an alkaline phosphatase conjugated anti-DIG antibody. The chemiluminescence signal was detected using the substrate for alkaline phosphatase (DIG-High Prime DNA Labeling and Detection Starter kit II, Roche, Warsaw, Poland) and the G:BOX imaging system (Syngene, Cambridge, UK).

qPCR-based analysis of TE copy number

The following primers were used:

- Cirt2: AATAATGGATGGGCATGGAA, CCAA GGTCCAAAGGCTGTAA;

- Cmut1: TAGCTCGGCCTCAACATTTCC, AGC GTTTTCTAGCACGAAAGC;

- Moa: GTCGTGGAGTACGACGTtATCA, GTC GTGTAGTAGCAACACTTCG.

qPCR reaction mixture contained: $2 \mu \mathrm{l}$ of $300 \mathrm{ng}$ DNA, $10 \mu \mathrm{l}$ of Fast SYBR Green Master Mix (Roche, Warsaw, Poland), $1 \mu \mathrm{l}$ of $20 \mu \mathrm{M}$ each primer and ultrapure DNase/RNase-free water to a final volume of $20 \mu$ l. All qPCR reactions were performed in triplicate using Light Cycler $^{\circledR} 480$ system (Roche, Warsaw, Poland). The following qPCR reaction conditions were: a single cycle at $95{ }^{\circ} \mathrm{C}$ for $10 \mathrm{~min}$ and 40 cycles of $15 \mathrm{~s}$ at $95{ }^{\circ} \mathrm{C}$ and $20 \mathrm{~s}$ at $62{ }^{\circ} \mathrm{C}$. PCR products were 
then heated at $95{ }^{\circ} \mathrm{C}$ for $15 \mathrm{~s}$, cooled at $60{ }^{\circ} \mathrm{C}$ for $1 \mathrm{~min}$ and heated at $95{ }^{\circ} \mathrm{C}$ for $15 \mathrm{~s}$. The relative copy number of $\mathrm{TE}$ sequences was analyzed using the LightCycler ${ }^{\circledR} 480$ Software using the basic relative quantification according to beta-actin gene copy number.

Analysis of chromosomal DNA breaks and replication intermediates (RIs)

For quantitative analysis of chromosomal DNA breaks and replication intermediates (RIs), single chromosome comet assay was used (Lewinska et al. 2014b). Preparation of agarose-embedded C. albicans DNA and PFGE separation of $C$. albicans DNA were performed as comprehensively described elsewhere (Lewinska et al. 2014b). After PFGE separation, $C$. albicans chromosomes were stained with ethidium bromide and bands were removed from the gel using a razor blade and single chromosome comet assay was conducted (Lewinska et al. 2014b). A total of 200 chromosomes per each sample strain triplicate were analyzed and the percentage of chromosomal DNA breaks and replication intermediates (RIs) were calculated as comprehensively described elsewhere (Adamczyk et al. 2016).

Multivariate data analysis

All obtained data were subjected to multivariate analysis using the Hierarchical Cluster Analysis (HCA) using ClustVis, a web tool for visualizing clustering of multivariate data (BETA) (https://biit.cs. ut.ee/clustvis/) (Metsalu and Vilo 2015). The following options were considered: pre-processing optionno transformation, clustering distance for rows and columns-Pearson correlation, clustering methods for rows and columns-average, tree ordering for rows and columns - tightest cluster first. A heat map was generated on the basis of growth rate, TE copy number, karyotype patterns, chromosomal DNA breaks and replication intermediates.

Statistical analysis

The mean values \pm SD were calculated on the basis of at least three independent experiments. Box and whisker plots were also considered. Statistical significance was evaluated using GraphPad Prism 5 using one-way ANOVA and Dunnett's test.

\section{Results}

Long-term culture-mediated changes

in morphology, growth rate and cell viability

In the present study, we have used a model of longterm culture of $C$. albicans cells (up to 90 days) and started the culture at relatively high cell density $\left(1 \times 10^{8}\right.$ cells $\left./ \mathrm{ml}\right)$ to mimic stationary phase conditions during chronological aging (CA) in yeast that is measured by monitoring the survival of a non-dividing population (Longo et al. 2012; Hu et al. 2013). However, rich medium (YPD medium) was considered instead of a classical CA medium, namely synthetic complete (SC) medium and YPD medium was replaced by a fresh one every $48 \mathrm{~h}$ of culture to avoid starvation and related stress responses that are common during CA in yeast (Longo et al. 2012; $\mathrm{Hu}$ et al. 2013). Moreover, we decided to start analyzing the parameters of long-term culture experiment after 14 days of culture because culturing the yeast cells in rich YPD medium is characterized by extended postdiauxic phase (up to 1 week) during which cells continue to grow slowly (Werner-Washburne et al. 1996; Longo et al. 2012). C. albicans cells of different ploidy were used, namely haploid reference strain 302 , diploid reference strain SC5314 and tetraploid reference strain T15 (Selmecki et al. 2008) and after 14, 28 and 90 days of culture, upon initial treatment with stress stimuli (fluconazole, hydrogen peroxide, hypochlorite) for 4 days, cells were taken for further analyses.

In general, yeast cell morphology (budding form, single-celled blastospore) was observed when cells were cultured in YPD medium (Fig. 1a).

Filamentous (pseudohyphal and hyphal) forms were almost not noticed (Fig. 1a). Typical shapes (spherical to oval) and sizes $(2-5 \times 3-7 \mu \mathrm{m})$ of $C$. albicans cells were observed during long-term culture (Fig. 1a). However, after 90 days of culture, cells became more elongated as judged by morphology analysis using imaging flow cytometry (Fig. 1b). A small fraction of non-spherical cells occurred (Fig. 1b) that was especially accented in tetraploid 
a

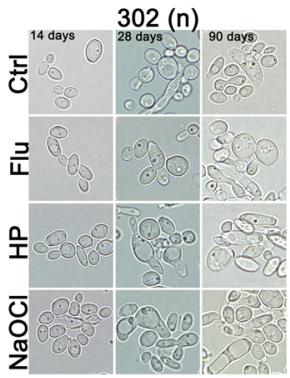

SC5314 (2n)

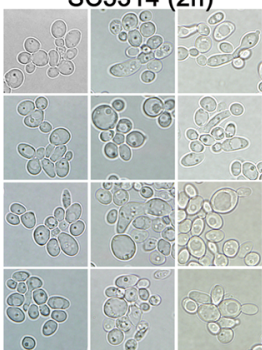

T15 (4n)

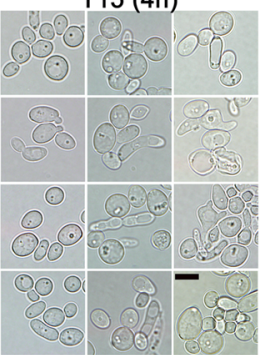

C

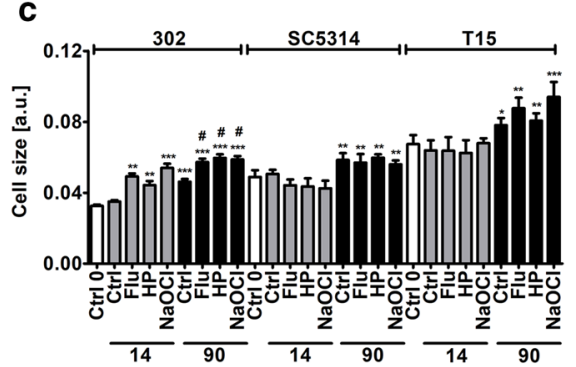

[d]

d

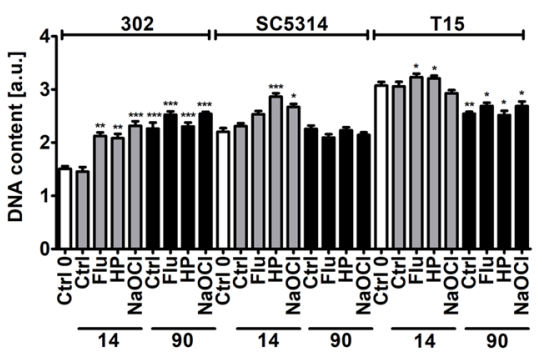

[d]

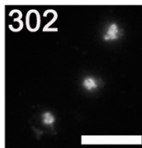

SC5314
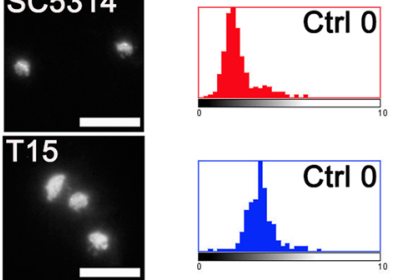

DNA content
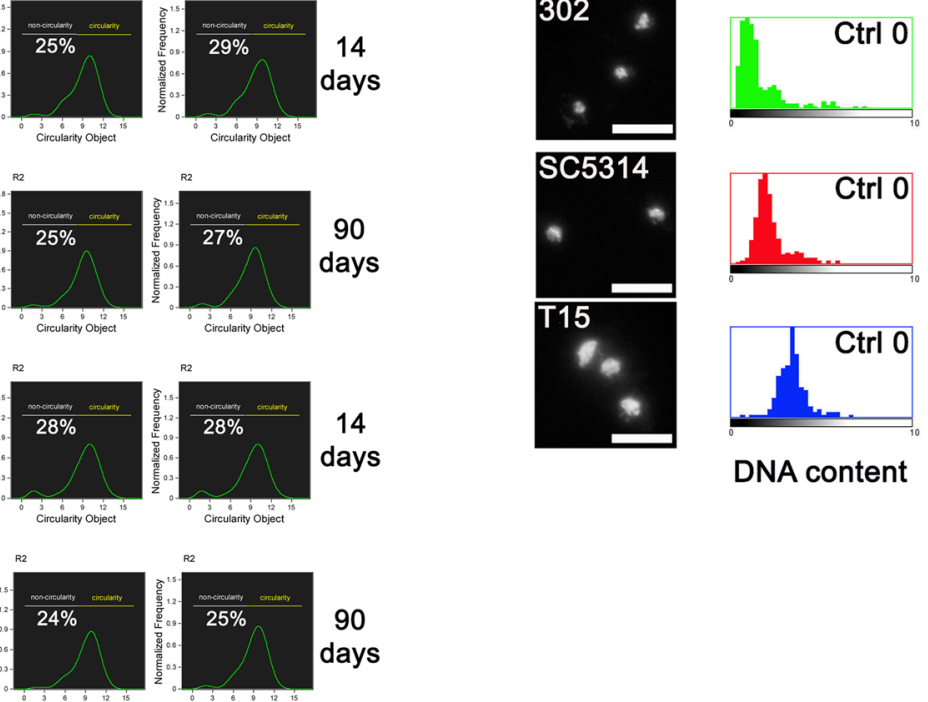

90

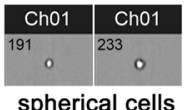

cells (an increase of 10\%) and in haploid cells (an increase of 8\%) (Fig. 1b). After 14 days of culture in control conditions, cell morphology was not affected compared to cell morphology at logarithmic phase of growth (overnight culture, time 0) (Fig. 1b). The effects of stress stimuli on cell morphology were limited (Fig. 1b). Elevated fraction of non-spherical cell population was observed in hypochlorite-treated haploid cells and fluconazole-treated tetraploid cells after 90 days of culture, namely an increase of $21 \%$ and $36 \%$ was noticed, respectively (Fig. 1b). Moreover, ploidy-dependent cell size variation and DNA 
४Fig. 1 Long-term culture-mediated Candida cell morphology $(\mathbf{a}, \mathbf{b})$, cell size (c) and DNA content $(\mathbf{d})$. a Cell morphology was analyzed using an Olympus BX61 fluorescence microscope equipped with a DP72 CCD camera and Olympus CellF software (Olympus). Representative microphotographs are shown. Scale bar $5 \mu \mathrm{m}$, objective $100 \times$. b Cell morphology (here shape) was analyzed using Amnis ${ }^{\circledR}$ FlowSight ${ }^{\circledR}$ imaging flow cytometer and IDEAS software (Merck Millipore). Two subpopulations of cells were considered, namely spherical cells (circularity) and non-spherical cells (non-circularity). Representative histograms and cell images are presented. The parameter circularity object was analyzed and normalized frequency was plotted against circularity object. 5000 cells per sample triplicate were analyzed and the percentage of cells of non-spherical cell subpopulation is presented. c ImageJ software (http://rsbweb.nih.gov/ij/) was used to analyze the cell size. Cell size was expressed as arbitrary units [a.u.]. d Fluorescence microscopy was adapted for DNA content analysis. DNA was visualized using DAPI staining. Representative microphotographs and data distribution (histograms) are shown. Scale bars $10 \mu \mathrm{m}$, objective 100x. ImageJ software (http://rsbweb.nih. gov/ij/) was used to analyze the nuclear DNA content. DNA content was expressed as arbitrary units [a.u.]. Bars indicate SEM, $\mathrm{n}=3 .{ }^{* * *} p<0.001,{ }^{* *} p<0.01,{ }^{*} p<0.05$ compared to control conditions after 14 days of culture, ${ }^{\#} p<0.05$ compared to control conditions after 90 days of culture (ANOVA and Dunnett's a posteriori test). Ctrl, control conditions; Ctrl 0, control conditions at the logarithmic phase of growth (overnight culture); Flu, fluconazole; $\mathrm{HP}$, hydrogen peroxide; $\mathrm{NaOCl}$; sodium hypochlorite; 14, 14 days of culture; 28, 28 days of culture; 90, 90 days of culture; 302, haploid strain; SC5314, diploid strain; T15, tetraploid strain

content analysis was considered (Fig. 1c, d). Of course, diploid and tetraploid cells were bigger than haploid cells after 14 days of culture in control conditions (Fig. 1c). After 90 days of culture, all three cell categories, namely haploid, diploid and tetraploid cells became bigger compared to $14 \mathrm{~d}$ culture in control conditions (Fig. 1c). Stress stimuli potentiated an increase in cell size of haploid cells both after 14 days as well as after 90 days of culture (Fig. 1c). As haploid strains of C. albicans are considered to be unstable and may autodiploidize (Hickman et al. 2013), we decided then to evaluate DNA content of haploid, diploid and tetraploid cells during long-term culture both in control conditions as well as upon stress agent stimulation (Fig. 1d). Of course, higher levels of DNA were observed in diploid and tetraploid cells compared to haploid cells after 14 days of culture (Fig. 1d). After 90 days of culture, DNA content of haploid cells was increased, whereas in tetraploid cells, DNA levels were decreased and DNA levels were not affected in diploid cells
(Fig. 1d). Thus, it seems that DNA content of haploid, diploid and tetraploid cells is more or less comparable after 90 days of culture in control conditions. Perhaps, this may reflect a tendency of Candida cells to buffer their genome to be more or less diploid in term of DNA content (Hickman et al. 2013, 2015). This may be a form of adaptation during long-term culture. Moreover, all three stress stimuli potentiated an increase in DNA content of haploid cells after 14 day of culture compared to untreated control (Fig. 1d). There were no changes in cell size and DNA content of haploid, diploid and tetraploid cells between time categories of 0 and 14 days (Fig. 1c, d). One should remember that long-term culture-mediated cell size may not correlate with DNA content of yeast cells of different ploidy because during prolonged treatment and aging increased cell size may be associated with increased vacuole size.

We have then addressed the question of whether growth rate and cell viability may be affected during long-term culture (Fig. 2).

There were no changes in growth rate of haploid 302 strain when grown up to 90 days both in control conditions and upon initial stimulation with stress agents for 4 days (Fig. 2a). In contrast, minor to moderate inhibitory effects were observed in diploid SC5314 and tetraploid T15 cells, respectively (Fig. 2a). Tetraploid cells were the most susceptible to long-term culture-mediated growth inhibition both in control conditions and upon treatment with stress agents (Fig. 2a). Cell viability of haploid, diploid and tetraploid strains was not affected during long-term culture in control conditions (Fig. 2b). Upon stimulation with stress agents, a slight increase in fraction of dead haploid and diploid cells was observed, especially after 90 days of culture (Fig. 2b). Similar effects were not noticed during long-term culture of tetraploid cells (Fig. 2b).

Long-term culture-promoted activation of transposable elements and changes in karyotype patterns

To study TE activity during long-term culture of $C$. albicans cells, we have selected three members from different TE groups, namely $C$. albicans transposon Cirt2 transposase (https://www.ncbi.nlm.nih.gov/ nuccore/AF205929.1), C. albicans retrotransposon LTR Moa, LTR associated with the transposon Tca13 
a
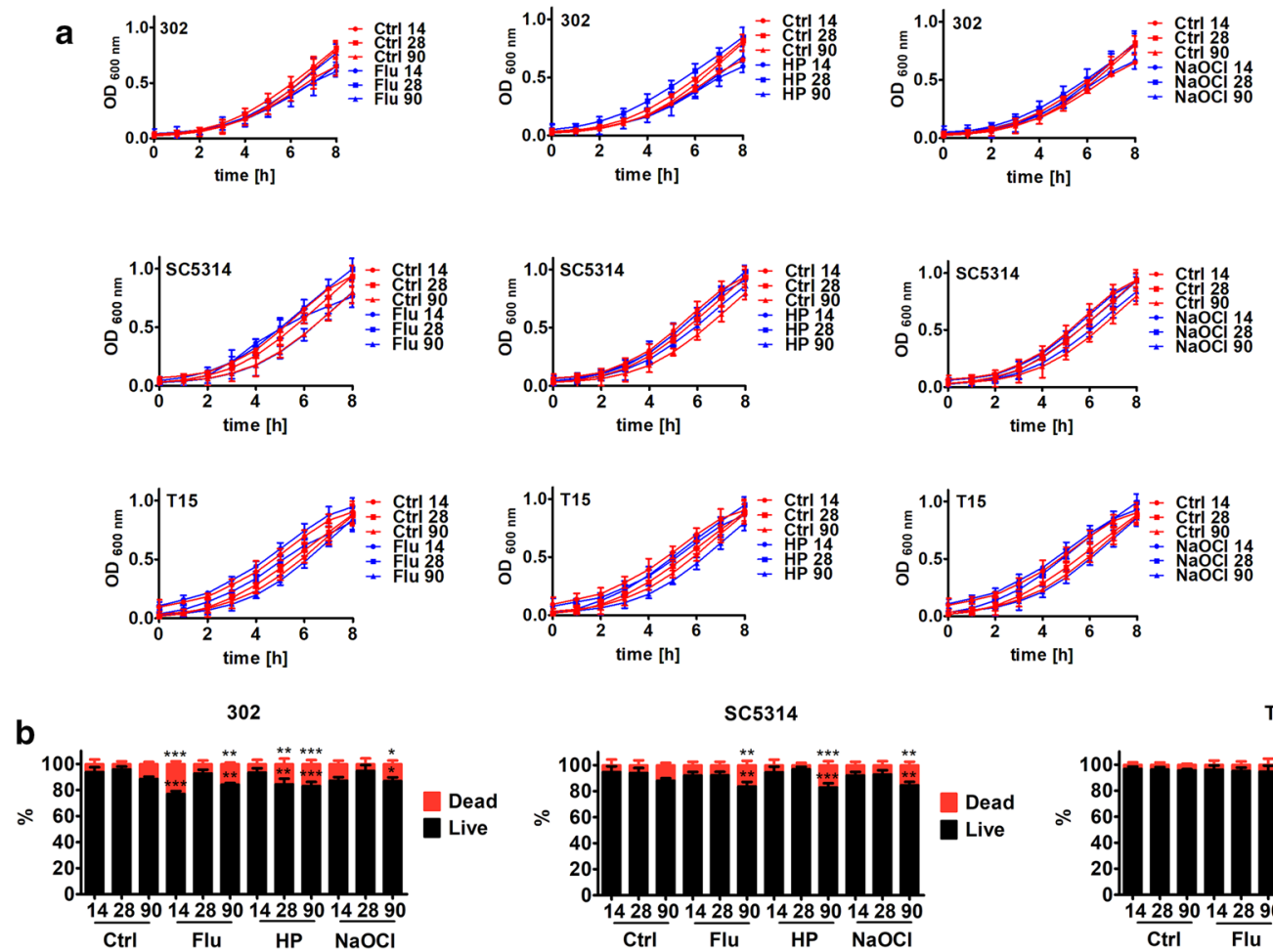

SC5314

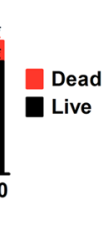

Fig. 2 Long-term culture-mediated growth rate (a) and cell viability (b). a After 14, 28 and 90 days of culture, C. albicans growth was monitored turbidimetrically at $600 \mathrm{~nm}$ in a microplate reader every $2 \mathrm{~h}$ during a $8 \mathrm{~h}$. b After 14, 28 and 90 days of culture, cell viability was estimated using a LIVE/ DEAD $^{\circledR}$ Yeast Viability Kit using the standard protocol according to the manufacturer's instructions. The percentage

(https://www.ncbi.nlm.nih.gov/nuccore/AF180291.1) (Goodwin and Poulter 2000) and Cmut1 mutator-like TE (MULE) of DNA transposons (class II elements) with homology to chromosome R sequence (https:// www.ncbi.nlm.nih.gov/nuccore/CP017630.1). For quantitative analysis of TE, copy number of Cirt2, Moa and Cmut 1 was investigated using qPCR analysis (Fig. 3).

Increased copy number of Cirt2 and Moa was observed during long-term culture of tetraploid cells and to a lesser extent augmented copy number of Cirt2 and Cmut1 was noticed in diploid cells (Fig. 3). The effect of culture duration was much more accented than the effect of stress inductors (Fig. 3).

CHEF-PFGE separation was then considered for karyotype profiling during long-term culture (Fig. 4a).

According to PFGE results, we were able to distinguish from seven to eight separate chromosomes that is due to the fact that chromosome $\mathrm{R}$ and

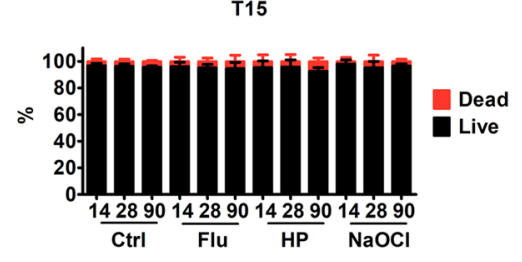

of live and dead cells is shown. Bars indicate SD, $\mathrm{n}=200$. $* * * p<0.001, * * p<0.01, * p<0.05$ compared to control conditions after 14 days of culture (ANOVA and Dunnett's a posteriori test). Ctrl, control conditions; Flu, fluconazole; HP, hydrogen peroxide; $\mathrm{NaOCl}$; sodium hypochlorite; 14,14 days of culture; 28, 28 days of culture; 90, 90 days of culture; 302 , haploid strain; SC5314, diploid strain; T15, tetraploid strain

chromosome I may migrate together (Fig. 4a). Indeed, it is widely accepted that $C$. albicans cells have eight pairs of chromosomal homologs (Chibana et al. 2000), but one should also remember that $C$. albicans species are characterized by a high genomic diversity (Magee and Magee 1987; Chibana et al. 2000). There were no changes in karyotype patterns during long-term culture in control conditions and after treatment with stress stimuli in diploid cells (Fig. 4a). Thus, the genome of SC5314 cells at chromosome level may be considered stable and not prone to changes in chromosome number (Fig. 4a). In contrast, after 90 days of culture upon initial treatment with fluconazole, hydrogen peroxide and hypochlorite, additional chromosome bands were observed in tetraploid cells as well as to a lesser extent after 90 days of culture in control conditions and after treatment with hypochlorite in haploid cells (Fig. 4a, at chromosome VI and VII positions, red arrows). The most accented 

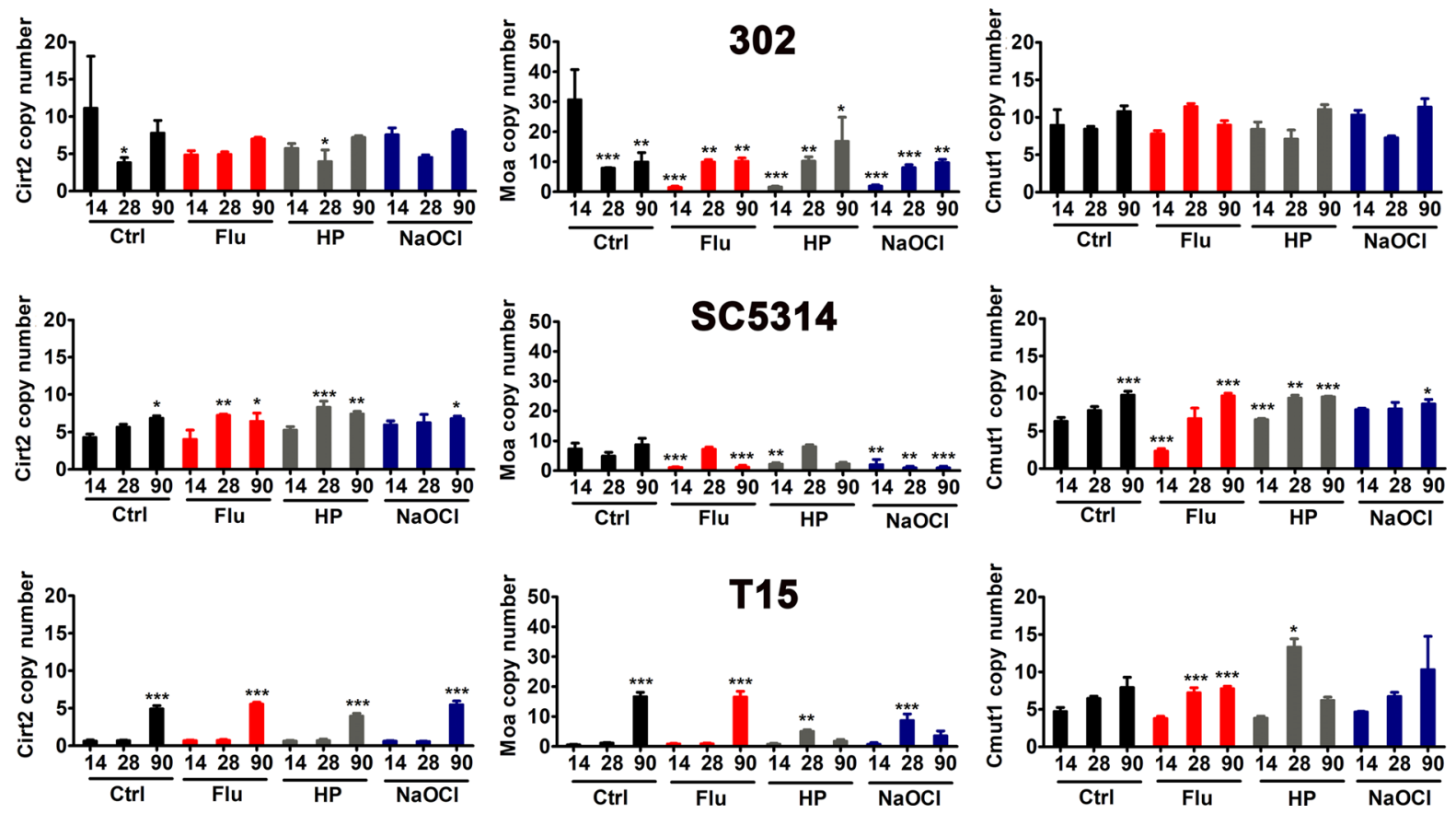

Fig. 3 Long-term culture-mediated changes in the copy number of selected transposable elements, namely Cirt2, Moa and Cmut1. qPCR reactions were performed in triplicate using Light Cycler $^{\circledR} 480$ system and relative copy number of TE sequences was analyzed using the LightCycler ${ }^{\circledR} 480$ Software using the basic relative quantification according to beta-actin gene copy number. Bars indicate $\mathrm{SD}, \mathrm{n}=3$. $* * * p<0.001$, ${ }^{* *} p<$

variability of karyotype profiles was correlated with the most pronounced increase in TE activity (here copy number) during long-term culture of tetraploid cells (Figs. 3, 4a). Thus, we decided then to analyze long-term culture mediated changes in chromosomal localization of TE (Fig. 4b). Cirt2 was detected on chromosome IV, Moa was detected on chromosomes I and VI and Cmut1 was detected on chromosome $\mathrm{R}$ (Fig. 4b, red arrowheads) that is in agreement with data provided at Candida genome database (www. candidagenome.org). However, no significant changes were found in their positions at chromosomes during long-term culture as judged by Southern blot analysis after CHEF-PFGE separation (Fig. 4b, red arrowheads). There were no correlations between the presence of additional bands (302 and T15 strains after 90 days of culture, Fig. 4a) and changes in TE chromosomal positions (Fig. 4b, red arrowheads). Southern blot analysis did not reveal increased levels of TE as already documented using qPCR analysis (Fig. 3). However, Southern blot is not as sensitive and reliable
$0.01, * p<0.05$ compared to control conditions after 14 days of culture (ANOVA and Dunnett's a posteriori test). Ctrl, control conditions; Flu, fluconazole; $\mathrm{HP}$, hydrogen peroxide; $\mathrm{NaOCl}$; sodium hypochlorite; 14, 14 days of culture; 28, 28 days of culture; 90, 90 days of culture; 302, haploid strain; SC5314, diploid strain; T15, tetraploid strain

quantitative analysis of TE as qPCR. There are also some limitations concerning data normalization. Typically, an equal amount of cells is used for spheroplast preparation but in the next step, the quantity of agarose-embedded DNA is not assayed. Moreover, spheroplast preparation from older cells with thicker cell wall may be less efficient compared to younger cells. Thus, Southern blot analysis was used only for analyzing the changes in TE chromosomal localization (qualitative analysis).

Long-term culture-induced chromosomal DNA breaks and replication intermediates

As increased TE activity may result in genetic instability, we have then considered single chromosome comet assay (Lewinska et al. 2014b) to analyze both general and specific susceptibility to DNA damage (here breaks) among C. albicans chromosomes during long-term culture (Fig. 5). 
a
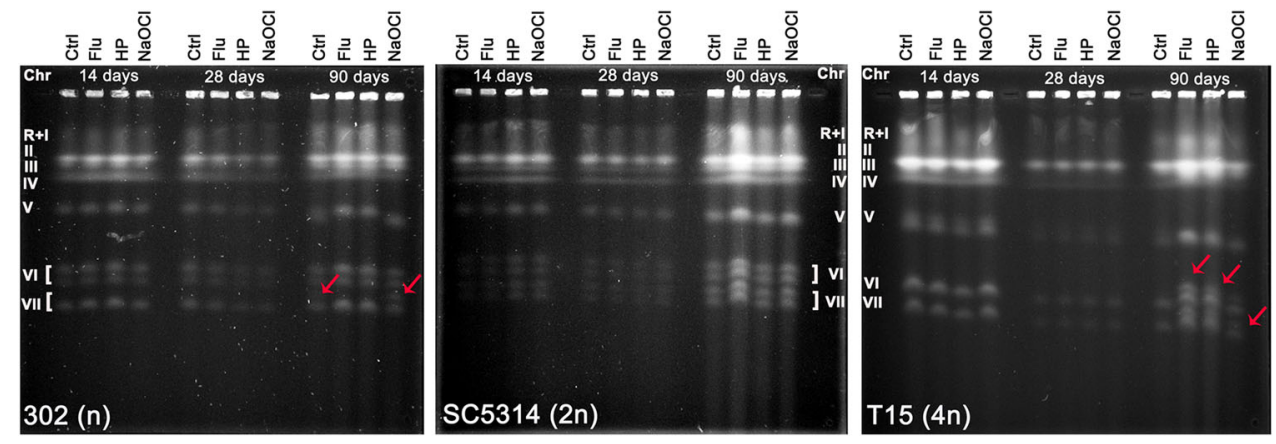

b

302

SC5314

T15
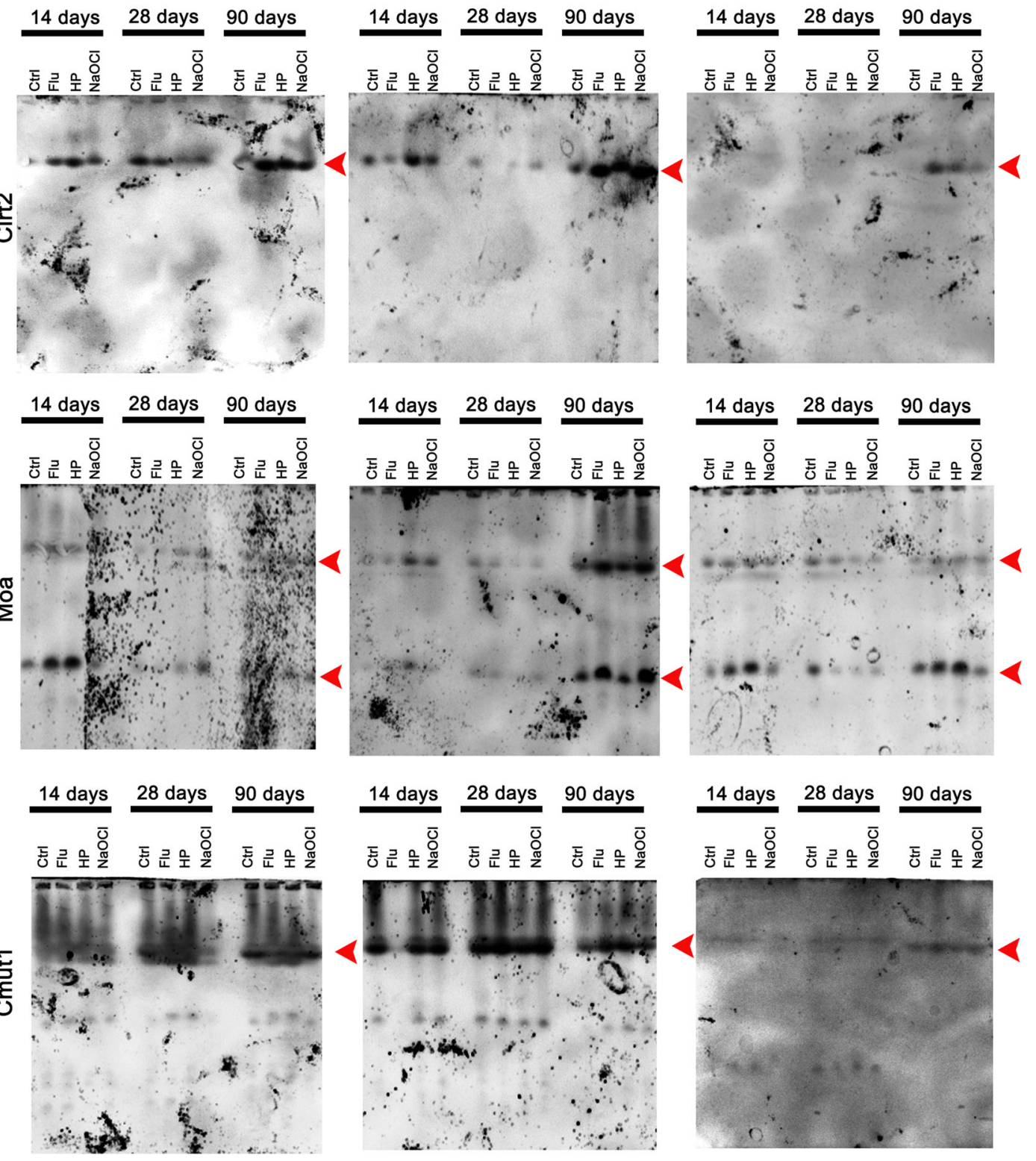
4Fig. 4 Long-term culture-mediated changes in karyotype patterns (a) and TE chromosomal localization (b). a Electrophoretic karyotyping (PFGE separation) of 302 haploid strain, SC5314 diploid strain and T15 tetraploid strain. Representative karyotype profiles are shown. Chromosomes were denoted as previously reported (Selmecki et al. 2005). Red arrows indicate additional chromosome bands that occurred after 90 days of culture. b Southern blot analysis of TE chromosomal localization. After PFGE separation, chromosomes were transferred onto a nylon membrane by capillary transfer, the membrane was hybridized to digoxigenin (DIG)labeled TE-specific probes and transposable elements (Cmut1, Cirt2, Moa, red arrowheads) were detected using an alkaline phosphatase conjugated anti-DIG antibody. The chemiluminescence signal was detected using the substrate for alkaline phosphatase (Roche) and the G:BOX imaging system (Syngene). Ctrl, control conditions; Flu, fluconazole; HP, hydrogen peroxide; $\mathrm{NaOCl}$; sodium hypochlorite; 14,14 days of culture; 28, 28 days of culture; 90, 90 days of culture; 302, haploid strain; SC5314, diploid strain; T15, tetraploid strain

As chromosome $\mathrm{R}$ and chromosome I may migrate together, DNA breaks and replication intermediates were analyzed in joined $\mathrm{R}+\mathrm{I}$ fraction and six separate fractions, namely chromosome II, chromosome III, chromosome IV, chromosome V, chromosome VI and chromosome VII fractions (Fig. 5a). After 90 days of long-term culture in control conditions, the most susceptible to DNA breaks were chromosomes III (haploid and tetraploid cells) and IV (diploid cells); an increase of about 13 to $14 \%$ in DNA breaks was observed, respectively (data not shown). Stress stimuli also promoted chromosomal DNA breaks, but various chromosomes were involved, e.g. chromosomes R, I and III in haploid cells, chromosomes VI and VII in diploid cells and chromosomes II, V and VII in tetraploid cells (data not shown). A joined analysis of chromosomal DNA breaks (Fig. 5b) revealed a statistically significant increase in chromosomal DNA breaks after 90 days of culture of haploid, diploid and tetraploid cells in control conditions and upon treatment with stress stimuli compared to control conditions after 14 days of culture (Fig. 5b). However, stress agents did not potentiate the levels of DNA breaks compared to control conditions after 90 days of culture (Fig. 5b). Moreover, we would like to verify if replication intermediates may accumulate during long-term culture that would be a sign of replication stress (Adamczyk et al. 2016). In contrast to long-term culture-induced chromosomal DNA breaks (Fig. 5b), an increase in replication intermediates was not observed after 90 days of culture (Fig. 5b). Stress agents also did not promote the accumulation of replication intermediates (Fig. 5b).

Multivariate data analysis

We have then considered hierarchical cluster analysis (HCA) using experimental data concerning growth rate, TE copy number, karyotype patterns, chromosomal DNA breaks and replication intermediates as a function of culture duration and treatment with stress agents (Fig. 6). Three C. albicans strains, namely haploid, diploid and tetraploid cells were analyzed separately (Fig. 6).

In a case of tetraploid T15 strain, all analyzed experimental parameters were grouped according to culture duration (14 days vs. 90 days) (Fig. 6). No effects of stress stimuli on data clustering were revealed (Fig. 6). Except of fluconazole treatment and 14 days of culture, similar observation was obtained for diploid SC5314 strain (Fig. 6). Again, no effects of stress stimuli on data clustering were noticed (Fig. 6). In contrast, in a case of haploid 302 strain, data clustering according to stress stimuli was revealed and the effect of culture duration was less evident (Fig. 6). This suggests that ploidy state of $C$. albicans strains may modulate adaptive responses during long-term culture, namely in diploid and tetraploid cells, changes in TE activity reflected culture duration, whereas in haploid cells, the effect of stress stimuli on TE activity was much more pronounced.

\section{Discussion}

In the present study, we have shown for the first time that the activity of Cirt2, Moa and Cmut 1 transposable elements (TEs) is elevated during long-term culture of C. albicans cells of different ploidy that is accompanied by changes in chromosome patterns (the occurrence of additional chromosome bands after 90 days of culture both in control conditions as well as after treatment with stress stimuli, namely fluconazole, hydrogen peroxide, hypochlorite) (Fig. 7).

Long-term culture-induced chromosomal DNA breaks were also observed, but the most accented increase in TE activity in T15 tetraploid cells did not correlate with the most pronounced increase in chromosomal DNA breaks in T15 tetraploid cells. In 
a
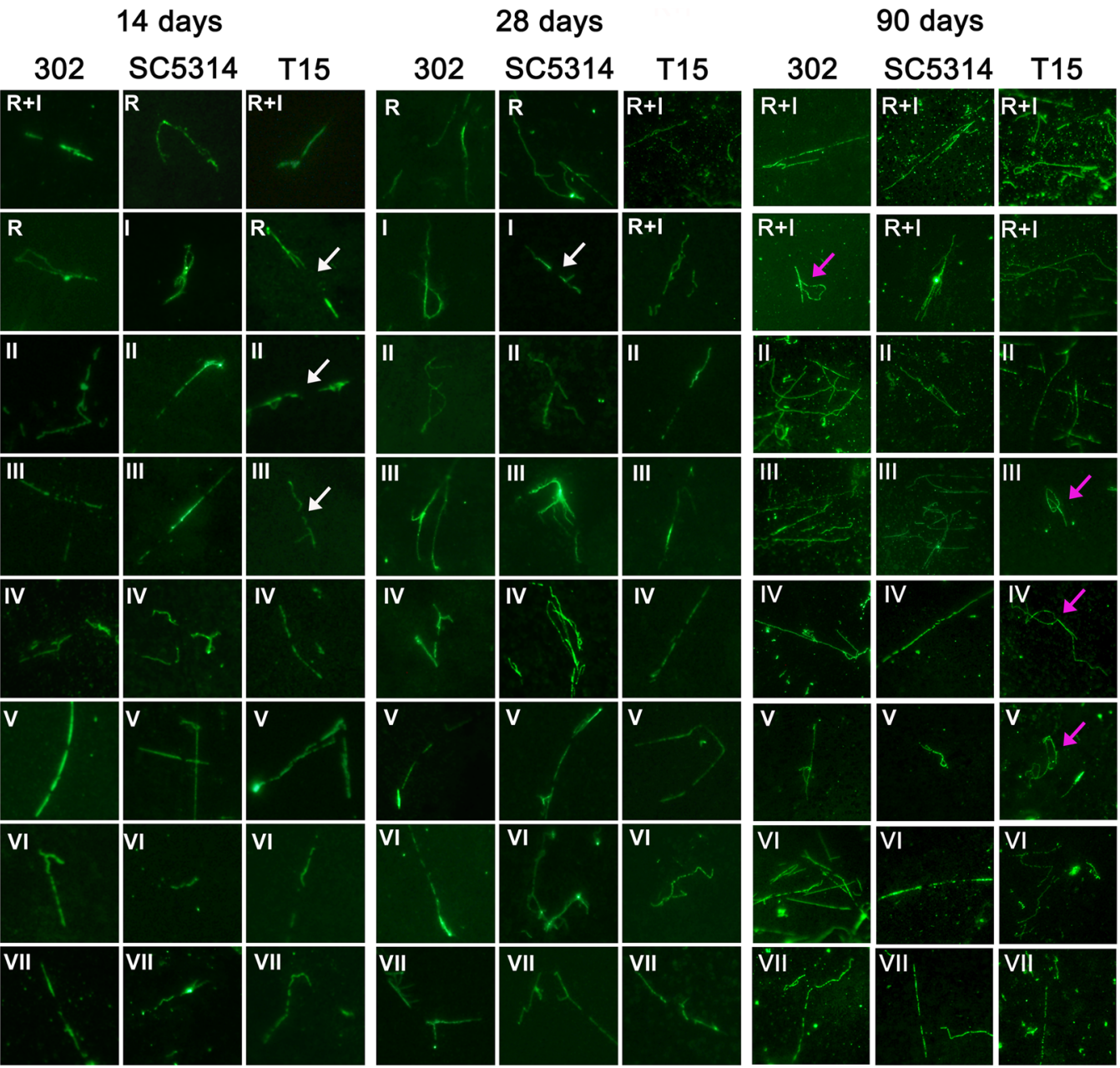

b
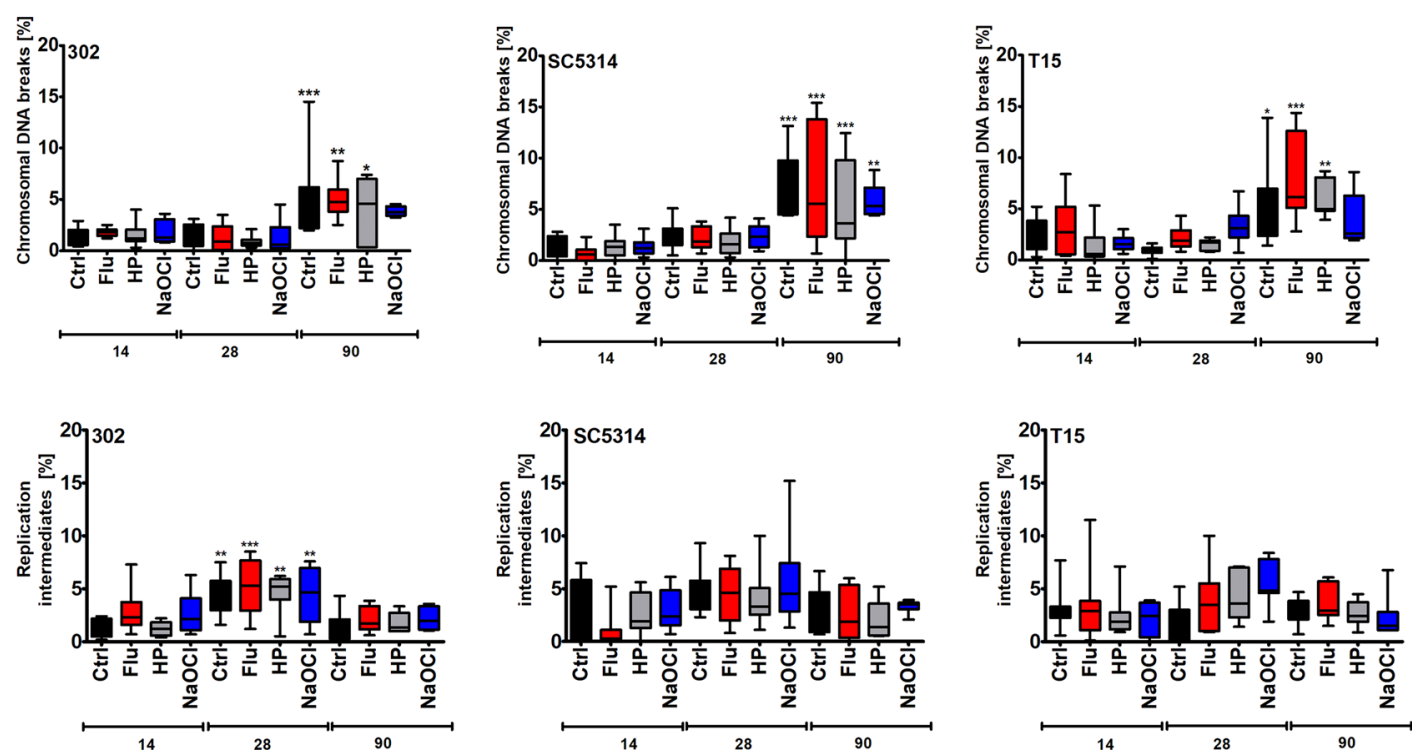
४Fig. 5 Long-term culture-induced chromosomal DNA breaks and replication intermediates. a After 14, 28 and 90 days of culture, chromosomal DNA breaks (white arrows) and replication intermediates (pink arrows) were revealed using single chromosome comet assay and an Olympus BX61 fluorescence microscope equipped with a DP72 CCD camera and Olympus CellF software. Briefly, C. albicans chromosomes were separated using PFGE and selected bands were cut using a razor blade. After electrophoresis, chromosomal DNA was stained with YOYO-1 stain solution (green). Representative microphotographs are shown. $\mathrm{R}+\mathrm{I}$; a joined fraction of chromosome R and chromosome I; II; chromosome II; III; chromosome III; IV; chromosome IV; V; chromosome V; VI; chromosome VI; VII; chromosome VII. b A total of 200 chromosomes per sample triplicate were analyzed and the percentages of chromosomal DNA breaks and replication intermediates were calculated. Box and whisker plots are shown, $\mathrm{n}=3$. ***p $<0.001, * * p<0.01, * p<0.05$ compared to control conditions after 14 days of culture (ANOVA and Dunnett's a posteriori test). Ctrl, control conditions; Flu, fluconazole; $\mathrm{HP}$, hydrogen peroxide; $\mathrm{NaOCl}$; sodium hypochlorite; 14,14 days of culture; 28,28 days of culture; 90,90 days of culture; 302, haploid strain; SC5314, diploid strain; T15, tetraploid strain

contrast, the levels of long-term culture-mediated chromosomal DNA breaks were comparable among C. albicans strains of different ploidy and diverse magnitude of increase in TE activity. Observed genomic changes at chromosomal level may promote genomic diversity and plasticity and drive cellular heterogeneity as an adaptive response during longterm culture of $C$. albicans cell populations.

According to our knowledge, there are no data on changes in the mobilome and genetic stability during long-term culture or replicative aging or chronological aging in C. albicans. However, it has been shown that retrotransposition is associated with genome instability during chronological aging in the budding yeast $S$. cerevisiae (Maxwell et al. 2011). Age-related loss of heterozygosity and chromosome loss were limited by mutations or treatments that decreased Ty1 retrotransposition and Ty 1 mobility was increased in old yeast cells, and new retro-mobility events were associated with chromosome rearrangements (Maxwell et al. 2011). The authors concluded that retrotransposition may contribute to genetic damage during aging in diverse organisms (Maxwell et al. 2011). Preferential retrotransposition in yeast mother cells has been also found to be correlated with increased genome instability during replicative aging in S. cerevisiae (Patterson et al. 2015). Age-related increase in the activity and mobility of transposable elements has been also reported in mammalian cells and tissues (De Cecco et al. 2013a, b; Colombo et al. 2018), however, no conclusive biological role(s) of these events have been provided. It has been observed that during normal aging several families of retrotransposable elements (RTEs) are transcribed in mouse tissues and in advanced age the expression culminates in active transposition (De Cecco et al. 2013b). The authors suggested that the mobilization of RTEs is likely to be an important contributor to the progressive dysfunction of aging cells (De Cecco et al. 2013b). The expression of transposable elements was also activated during replicative senescence and stress-induced senescence (5-azacytidine, hydrogen peroxide and adriamycin treatments) in human fibroblasts (Colombo et al. 2018). The authors concluded that the expression of TEs might play a role in immune mediated clearance of senescent cells (Colombo et al. 2018). More recently, it has been reported that during cellular senescence, LINE-1 retrotransposable elements became transcriptionally de-repressed and activated a type-I interferon (IFN-I) response that promoted age-associated inflammation (De Cecco et al. 2019).

Moreover, information on molecular mechanisms that regulate replicative lifespan (RLS) and chronological lifespan (CLS) or survival and adaptive responses during long-term culture of $C$. albicans is scarce and fragmentary (Fu et al. 2008; Li et al. 2011; Chen et al. 2012; Gil-Bona et al. 2016). SIR2 gene, a member of the sirtuin family of genes, has been recognized as a regulator of replicative lifespan in $C$. albicans (Fu et al. 2008). Increased SIR 2 gene dosage extended RLS, whereas knockout of SIR 2 gene caused a decrease of RLS (Fu et al. 2008). Replicative aging in clinical C. glabrata populations has been also investigated (Bouklas et al. 2017). Old C. glabrata cells were found to be more resistant to hydrogen peroxide and neutrophil killing, whereas young cells adhered better to epithelial cell layers and the virulence of old compared to younger C. glabrata cells was enhanced in the Galleria mellonella infection model (Bouklas et al. 2017). The authors concluded that the pathogenesis may be affected by the generational age distribution of the infecting $C$. glabrata population in a host and replicative aging may play an unanticipated role in the transition from a commensal to a pathogen state (Bouklas et al. 2017). Two proteins, namely Goalp, a regulator of the $C$. 

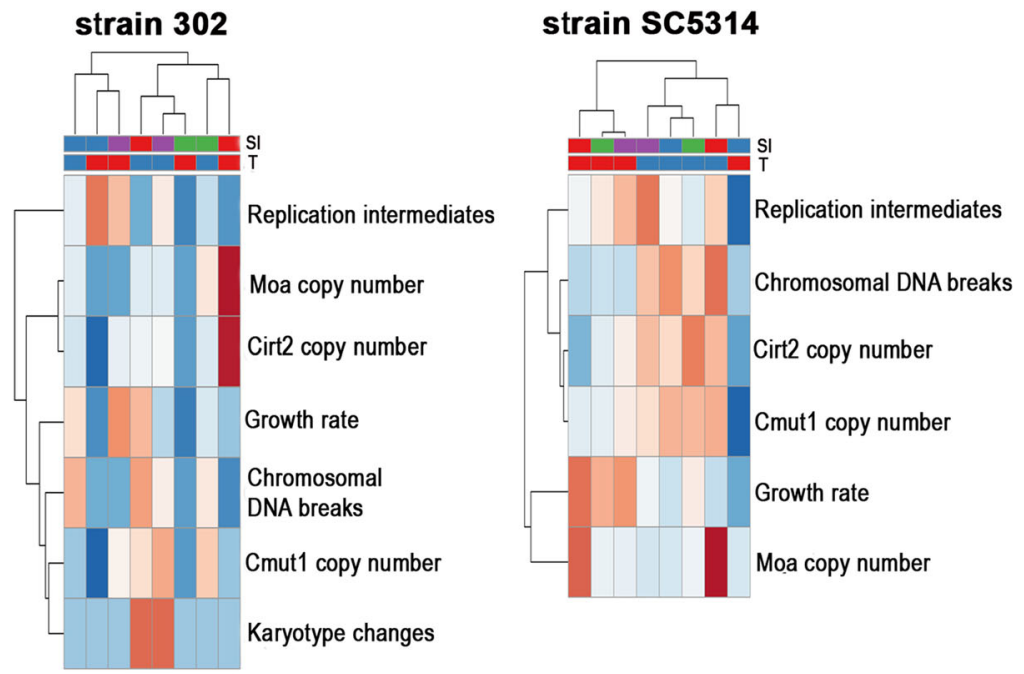

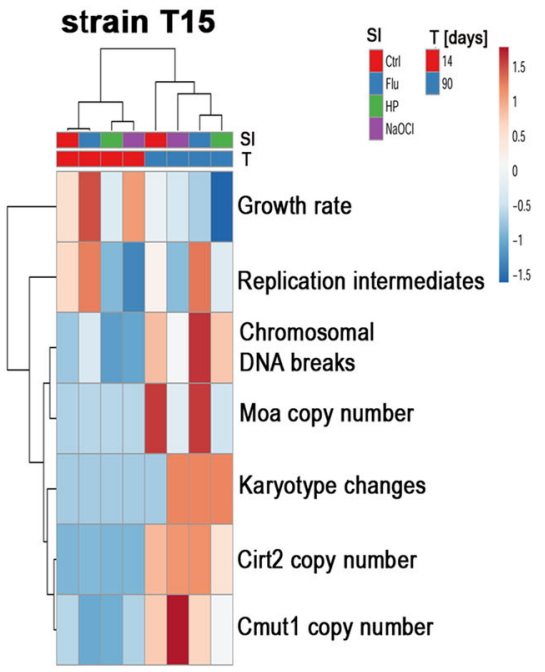

Fig. 6 A joined clustering analysis of growth rate, TE copy number, karyotype patterns, chromosomal DNA breaks and replication intermediates as a function of culture duration (14 days of culture versus 90 days of culture) and treatment with stress agents (fluconazole, hydrogen peroxide, hypochlorite). A heat map generated from growth rate, TE copy number, karyotype patterns, chromosomal DNA breaks and replication intermediates data is shown. Hierarchical clustering was created

albicans mitochondrial complex I, and the cell wall protein Ecm 33 have been documented to modulate CLS (Li et al. 2011; Gil-Bona et al. 2016). Deletion of GOA1 (growth and oxidant adaptation) gene resulted in oxidative stress, apoptosis and decreased CLS (Li et al. 2011) and caloric restriction restored CLS of the goal null mutant of C. albicans (Chen et al. 2012). C. albicans ecm33/ecm33 mutant devoid of a glycosylphosphatidylinositol-anchored protein involved in fungal cell wall integrity has been also shown to be hypersensitive to temperature, osmotic and oxidative stresses and to have a shortened CLS compared to wild-type strain (Gil-Bona et al. 2016). Oxidative stress is also implicated in chronological aging in other Candida species, namely C. glabrata as C. glabrata cells lacking both superoxide dismutases $\mathrm{Cu}, \mathrm{ZnSOD}$ (Sod1) and MnSOD (Sod2) showed a high rate of spontaneous mutation and decreased CLS compared to wild-type strain (Briones-Martin-del-Campo et al. 2015). During chronological aging, yeast cells are subjected to nutritional stress and oxidative stress due to the fact that yeast cells are maintained in a spent SC medium (Longo et al. 2012). Moreover, CLS is limited by the accumulation of acetic acid in a spent SC medium that may induce cell death itself and affect using ClustVis, a web tool for visualizing clustering of multivariate data (BETA) (https://biit.cs.ut.ee/clustvis/). Both rows and columns are clustered using correlation distance and average linkage for HCA analysis. Ctrl, control conditions; SI, stress inductors; Flu, fluconazole; HP, hydrogen peroxide; $\mathrm{NaOCl}$; sodium hypochlorite; $\mathrm{T}$, time; 14, 14 days of culture; 90, 90 days of culture; 302, haploid strain; SC5314, diploid strain; T15, tetraploid strain

CLS results (Burtner et al. 2009). Indeed, more recently, a number of potential artifacts that can affect CLS results obtained and their interpretation has been highlighted (Longo et al. 2012). Thus, to study longterm culture-mediated effects on $C$. albicans mobilome and genetic stability, we decided to use a rich YPD medium that was replaced every 2 days by a fresh one during 90 days of culture to limit nutritional stress- and oxidative stress-mediated effects on cell viability and related results. Indeed, no significant changes in cell viability were observed during 90 days of culture in control conditions (this study).

It has been repeatedly reported that the expression of transposable elements is induced by stress conditions in yeasts, plants, animals and human (McClintock 1984; Chen et al. 2003; Hashida et al. 2003; Todeschini et al. 2005; Hashida et al. 2006; Sehgal et al. 2007; Chenais et al. 2012; Jardim et al. 2015; Miousse et al. 2015; Morales et al. 2015). The mobility of TEs can be considered as a double-edged sword (Chenais et al. 2012). It may promote the occurrence of deleterious mutations, gene disruption and chromosome rearrangements, but on the other hand, transposition activity may be also beneficial and the mutational potential of TEs may contribute to the 
Fig. 7 The activity of selected transposable elements, namely Cirt2, Moa and Cmut 1 is increased during long-term culture of C. albicans cells of different ploidy that is accompanied by variations in karyotype patterns. Stress stimuli (fluconazole, hydrogen not potentiate TE activity. An increase in chromosomal DNA breaks was also noticed during long-term culture of $C$. albicans cells, however this was not correlated with elevated TE activity. Long-term culturemediated increase in TE activity and changes in karyotype profiles may in turn induce genomic diversity and cellular heterogeneity of $C$. albicans cell populations peroxide, hypochlorite) did

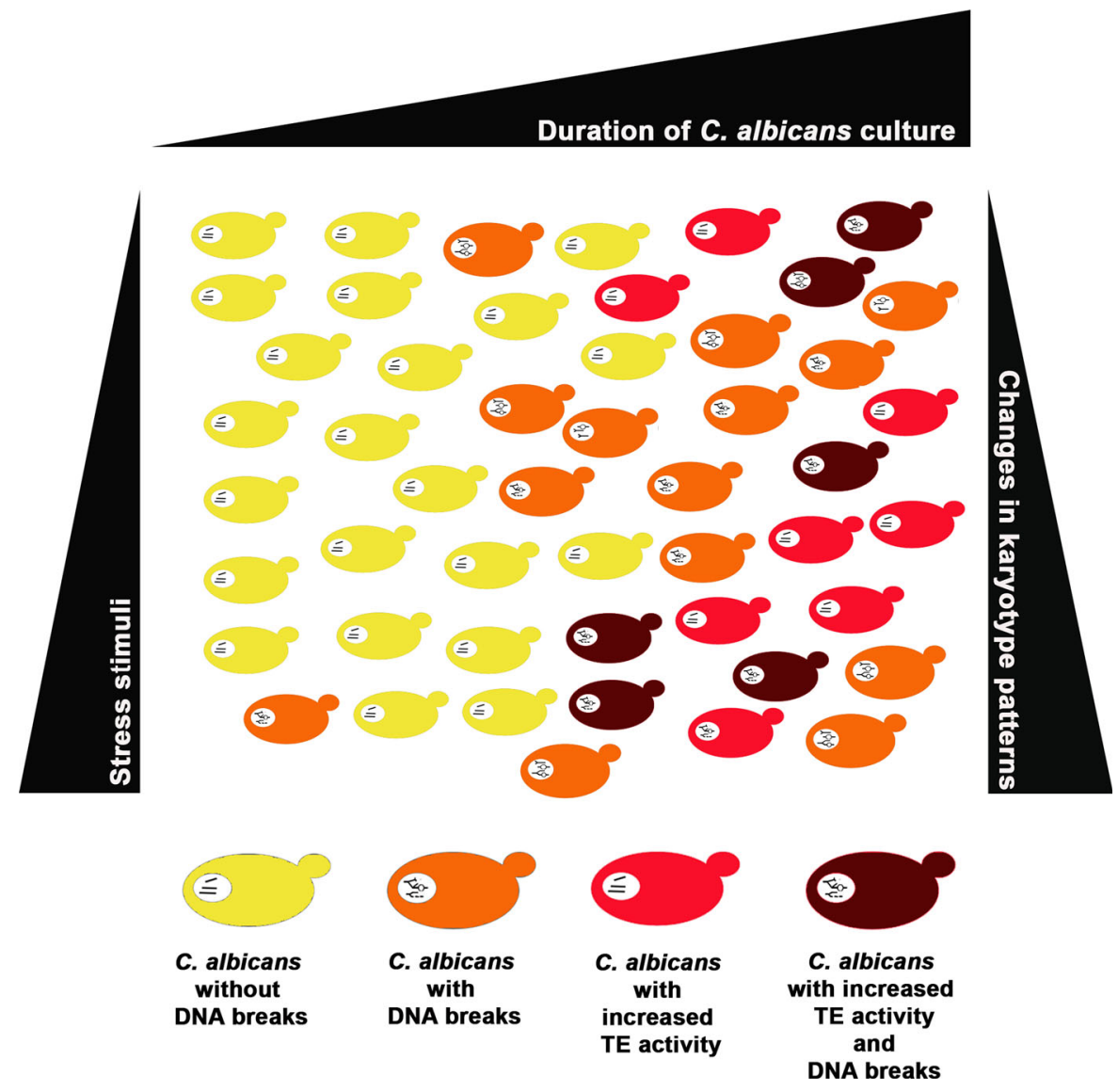

genetic diversity of organisms and genetic adaptations to stressful environments (Chenais et al. 2012). Indeed, transposon integration may enhance the expression of stress response genes and may drive adaptation to stress conditions (Feng et al. 2013; Esnault et al. 2019). The integration preference of Tf1, a long-terminal repeat retrotransposon in Schizosaccharomyces pombe, for the promoters of stress response genes has been documented (Feng et al. 2013) and the authors suggested that the ability of Tf1 to enhance the expression of these genes co-evolved to promote the survival of yeast cells under stress (Feng et al. 2013). In our experimental conditions, increased TE activity (increased TE copy number) was more related to culture duration (90 days) than initial stimulation with stress stimuli as stress stimuli did not potentate TE activity compared to control conditions after 90 days of long-term culture (this study). In general, this observation may reflect age-related changes in TE activity and associated mechanisms, namely the loss of histones or heterochromatin or aberrant RNAi pathway (Hu et al. 2014; Orr 2016; Drinnenberg et al. 2009). However, more studies are needed to reveal the molecular basis of long-term culture-mediated changes in TE activity in $C$. albicans.

Except of stable karyotype profile of diploid cells, affected TE activity correlated with the occurrence of additional chromosome bands in haploid and tetraploid cells both in control conditions and after treatment with stress agents that confirm the genomic plasticity of Candida species (Selmecki et al. 2010; Berman 2016; Todd et al. 2017) and may be a part of adaptive response during long-term culture. Haploid, diploid and tetraploid Candida cells can be observed as well as aneuploidy may be promoted (Perepnikhatka et al. 1999; Hickman et al. 2013; Selmecki et al. 2005) as a result of drug-induced genomic instability or as a selective advantage in the presence of the drug (Harrison et al. 2014). Variations in 
karyotype profiles and changes in ploidy state may drive phenotypic changes e.g., adaptations to stressful environments, host niches and antifungal drug treatments (Selmecki et al. 2010; Berman 2016). Several mechanisms that underlie the genomic diversity of $C$. albicans have been proposed, e.g., chromosome length polymorphism, reciprocal translocation at the major repeat sequence loci, chromosomal deletion and trisomy of individual chromosomes (Chibana et al. 2000). For example, azole resistance in C. albicans is accompanied by a specific segmental aneuploidy (an isochromosome that is a fusion of the two left arms of chromosome V (Selmecki et al. 2006) that resulted in the amplification of two fluconazole resistance genes ERG11 and TAC1 (Selmecki et al. 2008). Moreover, trisomy of chromosome R and trisomy of chromosome IV may also confer resistance to azole treatment in $C$. albicans (Li et al. 2015; Anderson et al. 2017).

In summary, an adaptive response during long-term culture of $C$. albicans cells of various ploidy states has been documented that is based on increased TE activity and changes in karyotype patterns. Long-term culture promoted chromosomal DNA breaks, however, genetic instability did not affect cell viability. Stress conditions (fluconazole, hydrogen peroxide and hypochlorite treatments) have less pronounced effect on TE activity than culture duration (90 days). Shifts in karyotype profiles may in turn promote genomic plasticity and cellular heterogeneity that may modulate cell fitness and lifespan and result in the selection of best adapted cells within a $C$. albicans cell population.

Acknowledgements We are indebted to Prof. Judith Berman (Department of Molecular Microbiology and Biotechnology, Tel Aviv University, Israel) for sharing with us haploid (302), diploid (SC5314) and tetraploid (T15) reference strains and for scientific inspiration to perform the experiments included in the current study. This work was supported by MINIATURA1 Grant Number 2017/01/X/NZ2/00049 from National Science Center (Poland).

Author contributions Conceived and designed the experiments: LP. Performed the experiments: LP EK KF BK MW. Analyzed the data: LP AL MW. Contributed reagents/materials/analysis tools: LP AL MW. Wrote the paper: AL LP.

\section{Compliance with ethical standards}

Conflicts of interest No potential conflicts of interest were disclosed.
Open Access This article is distributed under the terms of the Creative Commons Attribution 4.0 International License (http:// creativecommons.org/licenses/by/4.0/), which permits unrestricted use, distribution, and reproduction in any medium, provided you give appropriate credit to the original author(s) and the source, provide a link to the Creative Commons license, and indicate if changes were made.

\section{References}

Adamczyk J, Deregowska A, Panek A, Golec E, Lewinska A, Wnuk M (2016) Affected chromosome homeostasis and genomic instability of clonal yeast cultures. Curr Genet 62(2):405-418

Anderson MZ, Saha A, Haseeb A, Bennett RJ (2017) A chromosome 4 trisomy contributes to increased fluconazole resistance in a clinical isolate of Candida albicans. Microbiology 163(6):856-865

Berman J (2016) Ploidy plasticity: a rapid and reversible strategy for adaptation to stress. FEMS Yeast Res 16(3):fow020

Bouklas T, Alonso-Crisostomo L, Szekely T Jr, Diago-Navarro E, Orner EP, Smith K, Munshi MA, Del Poeta M, Balazsi G, Fries BC (2017) Generational distribution of a Candida glabrata population: resilient old cells prevail, while younger cells dominate in the vulnerable host. PLoS Pathog 13(5):e1006355

Briones-Martin-del-Campo M, Orta-Zavalza E, Canas-Villamar I, Gutierrez-Escobedo G, Juarez-Cepeda J, Robledo-Marquez K, Arroyo-Helguera O, Castano I, De Las Penas A (2015) The superoxide dismutases of Candida glabrata protect against oxidative damage and are required for lysine biosynthesis, DNA integrity and chronological life survival. Microbiology 161(Pt 2):300-310

Burns KH (2017) Transposable elements in cancer. Nat Rev Cancer 17(7):415-424

Burns KH, Boeke JD (2012) Human transposon tectonics. Cell 149(4):740-752

Burtner CR, Murakami CJ, Kennedy BK, Kaeberlein M (2009) A molecular mechanism of chronological aging in yeast. Cell Cycle 8(8):1256-1270

Chen D, Toone WM, Mata J, Lyne R, Burns G, Kivinen K, Brazma A, Jones N, Bahler J (2003) Global transcriptional responses of fission yeast to environmental stress. Mol Biol Cell 14(1):214-229

Chen H, Calderone R, Sun N, Wang Y, Li D (2012) Caloric restriction restores the chronological life span of the Goal null mutant of Candida albicans in spite of high cell levels of ROS. Fungal Genet Biol 49(12):1023-1032

Chenais B, Caruso A, Hiard S, Casse N (2012) The impact of transposable elements on eukaryotic genomes: from genome size increase to genetic adaptation to stressful environments. Gene 509(1):7-15

Chibana H, Beckerman JL, Magee PT (2000) Fine-resolution physical mapping of genomic diversity in Candida albicans. Genome Res 10(12):1865-1877

Colombo AR, Elias HK, Ramsingh G (2018) Senescence induction universally activates transposable element expression. Cell Cycle 17(14):1846-1857

De Cecco M, Criscione SW, Peckham EJ, Hillenmeyer S, Hamm EA, Manivannan J, Peterson AL, Kreiling JA, 
Neretti N, Sedivy JM (2013a) Genomes of replicatively senescent cells undergo global epigenetic changes leading to gene silencing and activation of transposable elements. Aging Cell 12(2):247-256

De Cecco M, Criscione SW, Peterson AL, Neretti N, Sedivy JM, Kreiling JA (2013b) Transposable elements become active and mobile in the genomes of aging mammalian somatic tissues. Aging (Albany NY) 5(12):867-883

De Cecco M, Ito T, Petrashen AP, Elias AE, Skvir NJ, Criscione SW, Caligiana A, Brocculi G, Adney EM, Boeke JD, Le O, Beausejour C, Ambati J, Ambati K, Simon M, Seluanov A, Gorbunova V, Slagboom PE, Helfand SL, Neretti N, Sedivy JM (2019) L1 drives IFN in senescent cells and promotes age-associated inflammation. Nature 566(7742):73-78

Drinnenberg IA, Weinberg DE, Xie KT, Mower JP, Wolfe KH, Fink GR, Bartel DP (2009) RNAi in budding yeast. Science 326(5952):544-550

Esnault C, Lee M, Ham C, Levin HL (2019) Transposable element insertions in fission yeast drive adaptation to environmental stress. Genome Res 29(1):85-95

Fabrizio P, Longo VD (2003) The chronological life span of Saccharomyces cerevisiae. Aging Cell 2(2):73-81

Feng G, Leem YE, Levin HL (2013) Transposon integration enhances expression of stress response genes. Nucleic Acids Res 41(2):775-789

Fu XH, Meng FL, Hu Y, Zhou JQ (2008) Candida albicans, a distinctive fungal model for cellular aging study. Aging Cell 7(5):746-757

Gil-Bona A, Reales-Calderon JA, Parra-Giraldo CM, MartinezLopez R, Monteoliva L, Gil C (2016) The cell wall protein Ecm33 of Candida albicans is involved in chronological life span, morphogenesis, cell wall regeneration, stress tolerance, and host-cell interaction. Front Microbiol 7:64

Goodwin TJ, Poulter RT (2000) Multiple LTR-retrotransposon families in the asexual yeast Candida albicans. Genome Res 10(2):174-191

Harrison BD, Hashemi J, Bibi M, Pulver R, Bavli D, Nahmias Y, Wellington M, Sapiro G, Berman J (2014) A tetraploid intermediate precedes aneuploid formation in yeasts exposed to fluconazole. PLoS Biol 12(3):e1001815

Hashida SN, Kitamura K, Mikami T, Kishima Y (2003) Temperature shift coordinately changes the activity and the methylation state of transposon Tam3 in Antirrhinum majus. Plant Physiol 132(3):1207-1216

Hashida SN, Uchiyama T, Martin C, Kishima Y, Sano Y, Mikami T (2006) The temperature-dependent change in methylation of the Antirrhinum transposon Tam 3 is controlled by the activity of its transposase. Plant Cell 18(1):104-118

Hickman MA, Zeng G, Forche A, Hirakawa MP, Abbey D, Harrison BD, Wang YM, Su CH, Bennett RJ, Wang Y, Berman J (2013) The 'obligate diploid' Candida albicans forms mating-competent haploids. Nature 494(7435):55-59

Hickman MA, Paulson C, Dudley A, Berman J (2015) Parasexual ploidy reduction drives population heterogeneity through random and transient aneuploidy in Candida albicans. Genetics 200(3):781-794

Hu J, Wei M, Mirisola MG, Longo VD (2013) Assessing chronological aging in Saccharomyces cerevisiae. Methods Mol Biol 965:463-472
Hu Z, Chen K, Xia Z, Chavez M, Pal S, Seol JH, Chen CC, Li W, Tyler JK (2014) Nucleosome loss leads to global transcriptional up-regulation and genomic instability during yeast aging. Genes Dev 28(4):396-408

Jardim SS, Schuch AP, Pereira CM, Loreto EL (2015) Effects of heat and UV radiation on the mobilization of transposon mariner-Mos1. Cell Stress Chaperones 20(5):843-851

Kaeberlein M (2010) Lessons on longevity from budding yeast. Nature 464(7288):513-519

Lewinska A, Macierzynska E, Grzelak A, Bartosz G (2011) A genetic analysis of nitric oxide-mediated signaling during chronological aging in the yeast. Biogerontology 12(4):309-320

Lewinska A, Miedziak B, Kulak K, Molon M, Wnuk M (2014a) Links between nucleolar activity, rDNA stability, aneuploidy and chronological aging in the yeast Saccharomyces cerevisiae. Biogerontology 15(3):289-316

Lewinska A, Miedziak B, Wnuk M (2014b) Assessment of yeast chromosome XII instability: single chromosome comet assay. Fungal Genet Biol 63:9-16

Li D, Chen H, Florentino A, Alex D, Sikorski P, Fonzi WA, Calderone R (2011) Enzymatic dysfunction of mitochondrial complex I of the Candida albicans goal mutant is associated with increased reactive oxidants and cell death. Eukaryot Cell 10(5):672-682

Li X, Yang F, Li D, Zhou M, Wang X, Xu Q, Zhang Y, Yan L, Jiang Y (2015) Trisomy of chromosome R confers resistance to triazoles in Candida albicans. Med Mycol 53(3):302-309

Lin SJ, Austriaco N (2014) Aging and cell death in the other yeasts, Schizosaccharomyces pombe and Candida albicans. FEMS Yeast Res 14(1):119-135

Longo VD, Shadel GS, Kaeberlein M, Kennedy B (2012) Replicative and chronological aging in Saccharomyces cerevisiae. Cell Metab 16(1):18-31

Magee BB, Magee PT (1987) Electrophoretic karyotypes and chromosome numbers in Candida species. J Gen Microbiol 133(2):425-430

Maxwell PH, Burhans WC, Curcio MJ (2011) Retrotransposition is associated with genome instability during chronological aging. Proc Natl Acad Sci USA 108(51):20376-20381

McClintock B (1984) The significance of responses of the genome to challenge. Science 226(4676):792-801

Metsalu T, Vilo J (2015) ClustVis: a web tool for visualizing clustering of multivariate data using principal component analysis and heatmap. Nucleic Acids Res 43(W1):W566-570

Miousse IR, Chalbot MC, Lumen A, Ferguson A, Kavouras IG, Koturbash I (2015) Response of transposable elements to environmental stressors. Mutat Res, Rev Mutat Res 765:19-39

Morales ME, Servant G, Ade C, Roy-Engel AM (2015) Altering genomic integrity: heavy metal exposure promotes transposable element-mediated damage. Biol Trace Elem Res 166(1):24-33

Mortimer RK, Johnston JR (1959) Life span of individual yeast cells. Nature 183(4677):1751-1752

Orr WC (2016) Tightening the connection between transposable element mobilization and aging. Proc Natl Acad Sci USA 113(40):11069-11070 
Patterson MN, Scannapieco AE, Au PH, Dorsey S, Royer CA, Maxwell PH (2015) Preferential retrotransposition in aging yeast mother cells is correlated with increased genome instability. DNA Repair (Amst) 34:18-27

Perepnikhatka V, Fischer FJ, Niimi M, Baker RA, Cannon RD, Wang YK, Sherman F, Rustchenko E (1999) Specific chromosome alterations in fluconazole-resistant mutants of Candida albicans. J Bacteriol 181(13):4041-4049

Potocki L, Depciuch J, Kuna E, Worek M, Lewinska A, Wnuk M (2019) FTIR and raman spectroscopy-based biochemical profiling reflects genomic diversity of clinical Candida isolates that may be useful for diagnosis and targeted therapy of Candidiasis. Int J Mol Sci 20(4):988

Sehgal A, Lee CY, Espenshade PJ (2007) SREBP controls oxygen-dependent mobilization of retrotransposons in fission yeast. PLoS Genet 3(8):e131

Selmecki A, Bergmann S, Berman J (2005) Comparative genome hybridization reveals widespread aneuploidy in Candida albicans laboratory strains. Mol Microbiol 55(5):1553-1565

Selmecki A, Forche A, Berman J (2006) Aneuploidy and isochromosome formation in drug-resistant Candida albicans. Science 313(5785):367-370

Selmecki A, Gerami-Nejad M, Paulson C, Forche A, Berman J (2008) An isochromosome confers drug resistance in vivo by amplification of two genes, ERG11 and TAC1. Mol Microbiol 68(3):624-641

Selmecki A, Forche A, Berman J (2010) Genomic plasticity of the human fungal pathogen Candida albicans. Eukaryot Cell 9(7):991-1008

Todd RT, Forche A, Selmecki A (2017) Ploidy variation in fungi: polyploidy, aneuploidy, and genome evolution. Microbiol Spectr. https://doi.org/10.1128/microbiolspec. FUNK-0051-2016

Todeschini AL, Morillon A, Springer M, Lesage P (2005) Severe adenine starvation activates Ty1 transcription and retrotransposition in Saccharomyces cerevisiae. Mol Cell Biol 25(17):7459-7472

Werner-Washburne M, Braun EL, Crawford ME, Peck VM (1996) Stationary phase in Saccharomyces cerevisiae. Mol Microbiol 19(6):1159-1166

Wicker T, Sabot F, Hua-Van A, Bennetzen JL, Capy P, Chalhoub B, Flavell A, Leroy P, Morgante M, Panaud O, Paux E, SanMiguel P, Schulman AH (2007) A unified classification system for eukaryotic transposable elements. Nat Rev Genet 8(12):973-982

Publisher's Note Springer Nature remains neutral with regard to jurisdictional claims in published maps and institutional affiliations. 